F. Brandonisio, M.P. Kennedy, F. Maloberti: "Observations on the Resolution and Tones in First Order Noise Shaping Time-to-Digital Converters"; 7th IEEE Conference on Ph.D. Research in Microelectronics and Electronics, PRIME 2011, Madonna di Campiglio, 3-7 July 2011, pp. 17-20.

(C)20xx IEEE. Personal use of this material is permitted. However, permission to reprint/republish this material for advertising or promotional purposes or for creating new collective works for resale or redistribution to servers or lists, or to reuse any copyrighted component of this work in other works must be obtained from the IEEE. 


\section{Observations on the Resolution and Tones in First Order Noise Shaping Time-to-Digital Converters}

\author{
Francesco Brandonisio \\ University College Cork and \\ Tyndall National Institute \\ Cork, Ireland
}

\author{
Michael Peter Kennedy \\ University College Cork and \\ Tyndall National Institute \\ Cork, Ireland
}

\author{
Franco Maloberti \\ Department of Electronics, \\ University of Pavia \\ Pavia, Italy
}

\begin{abstract}
In this paper we report some observations on the resolution and tones of First Order Noise Shaping LocalOscillator Based Time-to-Digital Converters (LO TDCs). We describe the architecture and governing equations of the LO TDC. We introduce equations to predict the resolution of the system "LO TDC plus moving average filter" and the frequency and amplitude of the largest amplitude tone in the spectrum of the TDC output when the input is constant. We describe briefly the block diagrams of a Matlab model of the LO TDC and its implementation on an FPGA. Finally, we compare analytical predictions of the amplitude and frequency of the largest tone in the spectrum with simulations and experimental results. The prediction of the powers and positions of the tones in the TDC output spectrum is fundamental for the design of the system in which the TDC has to be used.
\end{abstract}

\section{INTRODUCTION}

Precise measurements of time intervals between two events are fundamental in many applications that require time of flight measurements such as Positron Emission Tomography imaging [1] and radar systems [2], or clock related measurements, such as jitter measurements [3] and All-Digital-PLLs (ADPLLs) [4-6]. A Time-to-Digital Converter (TDC) measures an unknown time interval by counting how many reference time intervals it includes. The duration of the input time interval is typically determined by the edges of two digital signals, as shown in Fig. 1.

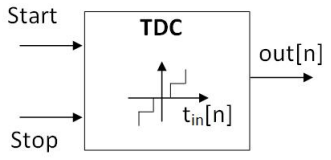

(a)

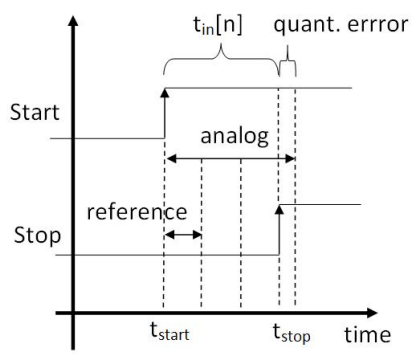

(b)
Fig. 1. Concept of a Time-to-Digital Converter.

There are two main kinds of reference time intervals: (i) the propagation delay of delay elements [7] and (ii) the oscillation period of oscillators [8]. Due to its finite resolution, a TDC introduces quantization noise on the output. Several techniques have been proposed in the literature to augment the resolution of TDCs and to reduce the quantization noise. These include Vernier architectures [9], interpolation techniques [8] and first order noise shaping TDCs [10].

In noise shaping TDCs, the resolution and signal to noise ratio at low frequencies are improved by pushing the power of the quantization noise to high frequencies, where it can later be removed by low-pass filtering. This feature makes noise shaping TDCs particularly interesting in ADPLLs, where the loop filter can be used to suppress the noise at high frequencies.

\section{Architecture of A First ORder Noise Shaping TDC}

In this work, we assume TDCs for measuring the phase error in ADPLLs. The phase error is associated with the duration of the time intervals between two consecutive edges of the reference oscillator and Digitally-Controlled-Oscillator (DCO) of an ADPLL. Thus, the edges of the reference oscillator and DCO define the time intervals that have to be measured at the measurement $n$ and those that are not interesting.

First order noise shaping of the quantization error is implemented when the measurement $n$ includes the quantization error of the previous measurement $(n-1)$.

In [10] and [11], a Gated Ring Oscillator-based TDC (GRO TDC) is used to implement first order noise shaping, as illustrated in Fig. 2(a). In the GRO TDC, the quantization error of every measurement is associated with the output charge of every stage of the GRO. The GRO is enabled only during the time intervals that have to be measured. First order noise shaping is achieved by preserving the state of the GRO between two consecutive measurements, as shown in Fig. 2(b). In fact, at the beginning of the current measurement the state of the GRO is the same of that at the end of the previous measurement, and consequently the quantization error of the previous measurement is included in the current one.

In [12], we proposed the first order noise shaping Local Oscillator based TDC (LO TDC) as an alternative to the GRO TDC. Figure 3(a) illustrates the block diagram of a simplified LO TDC, in which the time interval that has to be measured is the period of an input oscillator. In the LO TDC, the local oscillator is never disabled and the time intervals to be measured are consecutive. The value of the output charge of the LO at the beginning of the measurement $n$ is equal 
(a)

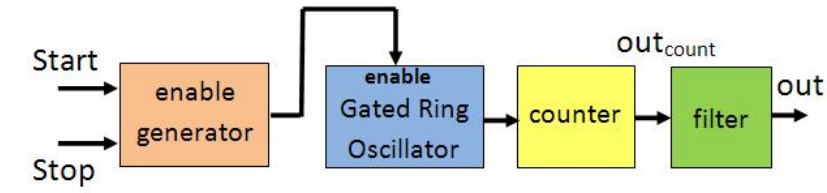

(b)

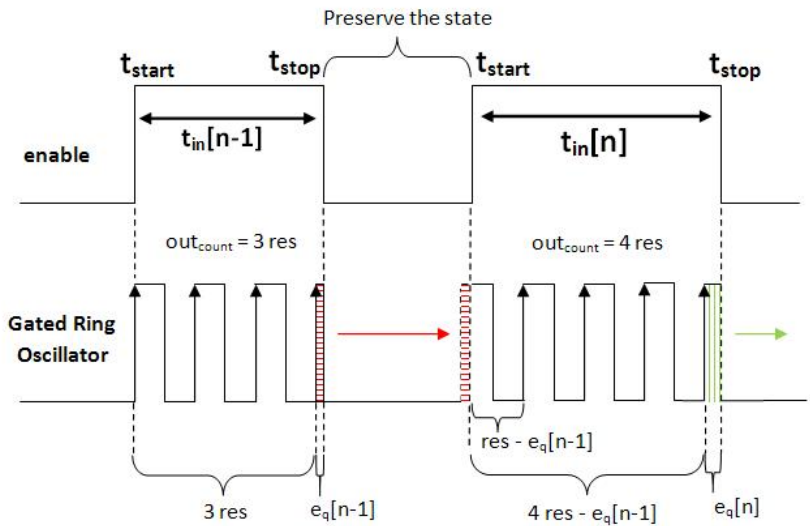

Fig. 2. Block (a) and timing (b) diagrams of a first order noise shaping GRO based TDC with filter.

to the value of the output charge of the end of the previous measurement $n-1$, by continuity, as shown in Fig. 3(b).

In a real GRO-TDC, the resolution of every measurement is not constant, because of the transient behavior of the GRO. By contrast, in the LO-TDC, the oscillator keeps running and there are no issues related to the transient behavior of the LO.

(a)

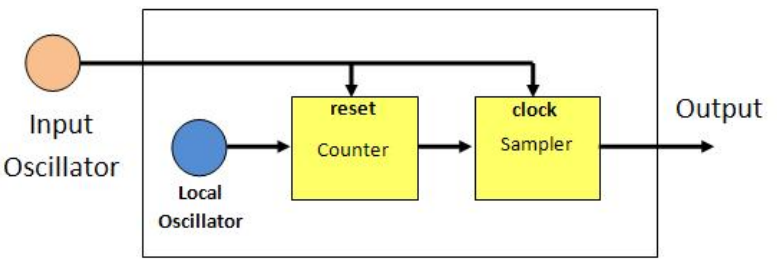

(b)

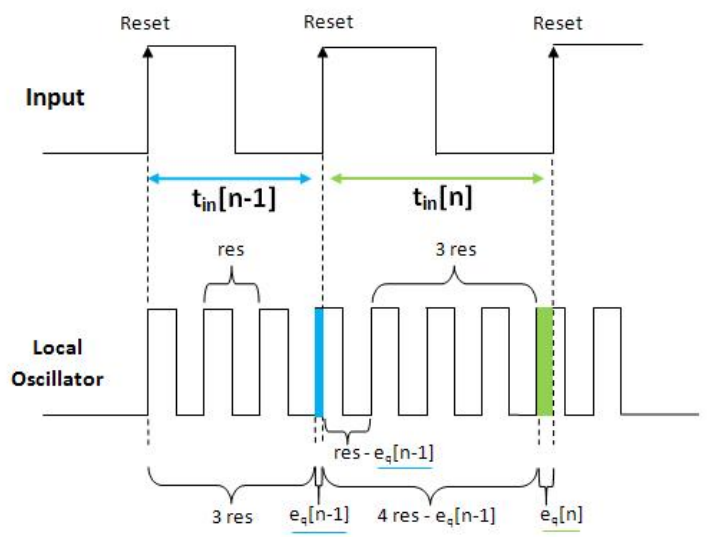

Fig. 3. Block (a) and timing (b) diagrams of the simplified LO TDC.

\section{Governing Equations and Matlab Model}

The simplified LO and GRO based TDCs are described by the same input-output equation, namely:

$$
\operatorname{out}[n]=\text { res } \cdot \text { floor }\left(\frac{t_{\text {in }}[n]-e_{q}[n-1]}{r e s}\right) .
$$

where $t_{i n}[n]$ is the time interval that has to be measured, res is the resolution, the floor $(\cdot)$ operator rounds to the closest integer less than the argument, and $e_{q}[n]$ is the quantization error at step $n$. The quantization error $e_{q}[n]$ is given by:

$e_{q}[n]=r e s \cdot$ floor $\left(\frac{t_{i n}[n]-e_{q}[n-1]}{r e s}\right)-t_{i n}[n]+e_{q}[n-1]$.

Figure 4 shows the block diagram of the model of the LOTDC in Fig. 3(a) that we have implemented in Matlab.

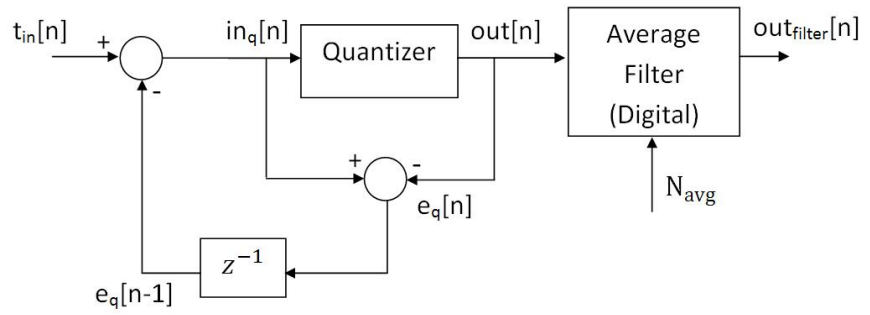

Fig. 4. Block diagram of the Matlab model of the first order noise shaping LO TDC with a moving average filter

Equations (1) and (2) can be derived from the block diagram in Fig. 4. In this work, we assume that $t_{i n}[n]$ is constant. As can be deduced from Eqs. (1) and (2), when the input of a first order noise shaping TDC with resolution res is a constant $T_{i n}$, the TDC output can assume only two values out $t_{1}=$ res floor $\left(T_{\text {in }} /\right.$ res $)$ and out $_{2}=$ res floor $\left(T_{\text {in }} /\right.$ res $)+1$, that are the two integer multiples of res closest to $T_{i n}$.

When the input is constant, the TDC output signal changes between out 1 and out ${ }_{2}$ with an average period $\overline{K_{1}}$. From Equations (1) and (2) it can be shown that:

$$
\overline{K_{1}}=\frac{r e s}{\operatorname{coef}},
$$

when coef $\leq 0.5$ and

$$
\overline{K_{1}}=\frac{\text { res }}{\bmod (\text { res }, \text { coef })},
$$

when coef $>0.5$, where coef $=\bmod \left(T_{\text {in }}\right.$, res $)$ and $\bmod (\cdot)$ and $\bmod (A, B)$ is the modulo operator with dividend $A$ and divisor $B$. Even though the average value of several periods of the TDC output signal is $\overline{K_{1}}$, a single period can be equal only to $\operatorname{ceil}\left(\overline{K_{1}}\right)$ or $\operatorname{ceil}\left(\overline{K_{1}}\right)-1$, where the $\operatorname{ceil}(\cdot)$ operator rounds to the closest integer greater than the argument. 


\section{Resolution And TONES}

A new resolution res $^{\prime}$ can be defined for a first order noise shaping TDC with resolution res plus a moving average filter. The main advantage of first order noise shaping of the quantization error is that the resolution of the system "first order noise shaping TDC plus filter" can be much higher than res. It can be proven by calculating the average value of the TDC output from Eqs. (1) and (2) that the average value of the output signal of the TDC is equal to the input signal when the input is constant. A digital moving average filter with window size equal to $N_{\text {avg }}$ samples can be used to process the output of the first order noise shaping TDC to obtain a digital measure of the input signal. The filter suppresses the high frequency components too. The output of the filter is given by:

$$
\text { out }_{\text {filt }}[n]=\frac{1}{N_{\text {avg }}} \sum_{i=n-N_{\text {avg }}+1}^{n} i n_{f i l t}[i],
$$

It can be proven from Eqs. (1), (2) and (5) that the resolution of the whole system "first order noise shaping TDC plus filter" is equal to res' for a constant input, where:

$$
r e s^{\prime}=\frac{r e s}{N_{a v g}} \text {. }
$$

In Eq. (6), res is the resolution of the first order noise shaping TDC, namely one period of the oscillator, and $N_{\text {avg }}$ is the number of samples used in the moving average filter. Figure 5 shows the input-output characteristics of the system "LO TDC plus filter" obtained with Matlab simulations of the system "LO TDC plus filter", when res $=1$ and $N_{\text {avg }}=5,10$ and 100, respectively. The size of the quantization step of the characteristics decreases when $N_{\text {avg }}$ increases, as predicted by Eq. (6). Therefore, the main advantage of the first order noise shaping of the quantization error is that the resolution of the system "first order noise shaping TDC plus filter" can be increased linearly by increasing the number of the samples of the moving average filter. Extra resolution can be obtained by a cascade of two or more moving average filters.

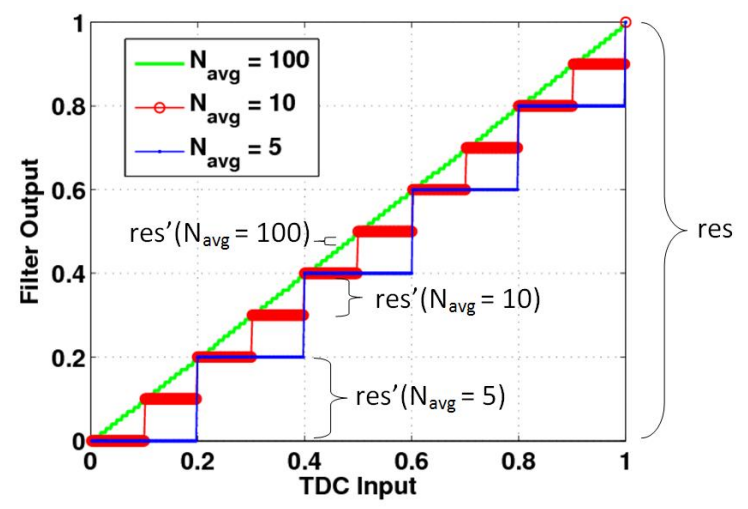

Fig. 5. Simulated input-output characteristics of the system "LO TDC plus filter" with res $=1$ and $N_{\text {avg }}=5,10,100$.

However, the spectrum of the output signal of a first order noise shaping TDC exhibits tones when the input is constant.
The positions in the spectrum and the powers of the tones are dependent on the input. These tones are the main drawback of first order noise shaping of the quantization error. The TDC output signal can be low pass filtered to suppress the extra spectral components introduced by first order noise shaping. The prediction of the powers and positions of the tones is fundamental for the design of a low pass filter. In this work, we focused on predictions of the frequency and amplitude of the largest amplitude component in the spectrum of a first order noise shaping TDC. It is possible to show from Eqs. (1)(4) that the amplitude of the largest amplitude component in the spectrum of the TDC output signal cannot be greater than $P_{\max }$, where:

$$
P_{\max }=\left(\frac{r e s}{\operatorname{ceil}\left(\overline{K_{1}}\right)}\right)^{2} .
$$

In Eq. (7), the ceil( $\cdot)$ operator rounds to the closest integer greater than the argument. From Eqs. (1) - (4) we can derive that the frequency of the largest amplitude component in the the spectrum of the TDC output signal is given by:

$$
\text { freq }_{\max }=\text { coef }
$$

when coef $\leq$ res/2; and

$$
f r e q_{\max }=r e s-\mathrm{coef},
$$

when coef $>$ res $/ 2$.

Equations (8) and (9) are in agreement with Eq. (2.26) in [13].

\section{EXPERIMENTAL SETUP AND RESUlts}

We realized the LO TDC on a Virtex-5 LX Xilinx Protoboard FF676. Figure 6 shows the preliminary experimental setup that we used to test the LO TDC.

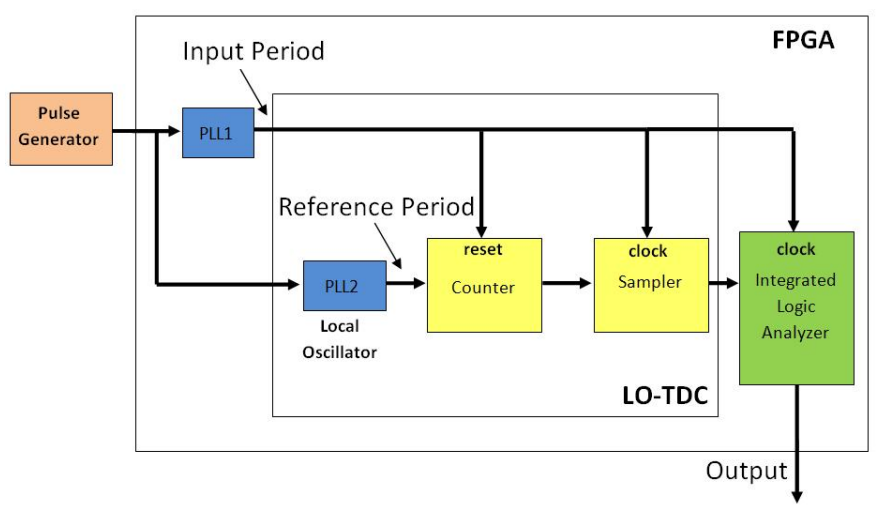

Fig. 6. block diagram of the LO TDC implemented on a FPGA.

An Agilent pulse generator $81130 \mathrm{~A}$ provides the clock to the board at $2 \mathrm{MHz}$. The on-board PLLs, labeled PLL1 and $P L L 2$ in Fig. 6, are used to generated the input and reference signals, respectively. The period of $P L L 2$, which is equal to about $500 \mathrm{~ns}$, is the resolution res of the LO TDC. The period of the output of PLL1 is the time interval that 
has to be measured. An on-board Integrated Logic Analyzer (ILA) samples the output of the LO TDC. Xilinx ChipScope Analyzer 11.1 is used to access to the data of the ILA. We measured the output signal of the LO based TDC for several values of the period of $P L L 1$, included in the interval from $24 \mathrm{~ns}$ to $455 \mathrm{~ns}$. The power spectral density of the output data of the TDC was calculated and processed in Matlab for every input signal in order to determine the frequency and the amplitude of largest amplitude component. Figures 7 and 8 show a comparison between predictions by means of Eqs. (7) - (9), Matlab simulations and measurements on the LO FPGA.

As can be seen in Fig. 7, the values of the amplitude of the largest tone in the spectra of the simulated and measured TDC output signals are less than $P_{\max }$ for every coef. There is good agreement between predicted, simulated and measured values of the frequency of the largest amplitude tone in the spectrum of the TDC output signal, as illustrated in Fig. 8.

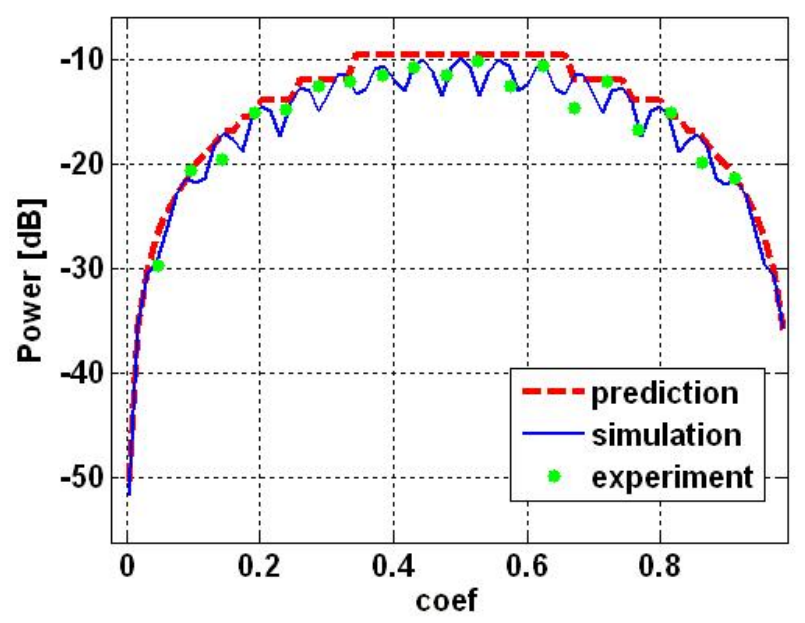

Fig. 7. Power of the largest amplitude component in the spectrum of the output signal of a first order noise shaping TDC with resolution res $=1$, measured, simulated and predicted analytically.

\section{CONCLUSIONS}

In this paper we have reported some observations on the resolution and tones of First Order Noise Shaping LocalOscillator Based Time-to-Digital Converters (LO TDCs). We have described the architecture and governing equations of the LO TDC. We have introduced equations to predict the resolution of the system "LO TDC plus moving average filter" and the frequency and amplitude of the largest amplitude tone in the spectrum of the TDC output when the input is constant. We illustrated briefly the block diagrams of a Matlab model and of an implementation on an FPGA of the LO TDC. The analytical predictions show good agreement with simulated and experimental results.

\section{ACKNOWLEDGEMENTS}

This work is supported in part by SFI under grant 08/IN1/1854 and by FIRB, Italian National Program, Project

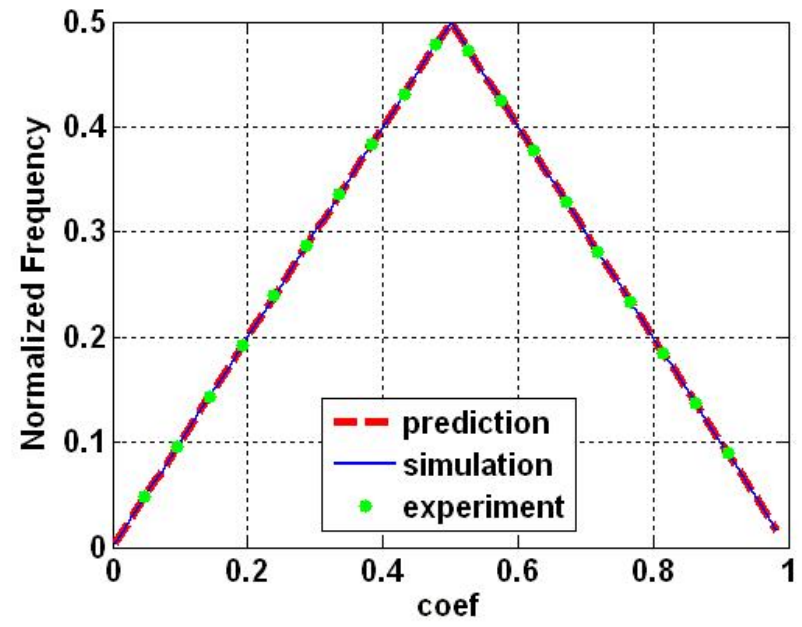

Fig. 8. Frequency of the largest amplitude component in the spectrum of the output signal of a first order noise shaping TDC with resolution res $=1$, measured, simulated and predicted analytically.

RBAP06L4S5.

\section{REFERENCES}

[1] A. S. Yousif, J. W. Haslett, "A Fine Resolution TDC Architecture for Next Generation PET Imaging," IEEE trans. on nuclear science, vol. 54, no. 5 , Oct. 2007

[2] I. Nissinen, J. Kostamovaara, "On-Chip Voltage Reference-Based Timeto-Digital Converter for Pulsed Time-of-Flight Laser Radar Measurements," IEEE trans. on instrumentation and measurement, vol. 58, no. 6, Jun. 2009.

[3] J.-C. Hsu, C. Su, "BIST for Measuring Clock Jitter of Charge-Pump Phase-Locked Loops," IEEE trans. on instrumentation and measurement, vol. 57, no. 2, Feb. 2008.

[4] R. B. Staszewski, P, T. Balsara, "Phase-Domain All-Digital Phase-Locked Loop," IEEE trans. on circuits and systems-II: express briefs, vol. 52, no. 3, Mar. 2005.

[5] C.-M. Hsu, M. Z. Straayer, M. H. Perrott, "A Low-Noise Wide-BW 3.6-GHz Digital $\triangle \Sigma$ Fractional-N Frequency Synthesizer With a NoiseShaping Time-to-Digital Converter and Quantization Noise Cancellation," IEEE J. Solid-State Circ., vol. 43, no. 12, pp. 2776-2786, Dec. 2008.

[6] E. Temporiti, C. Weltin-Wu, D. Baldi, R. Tonietto, F. Svelto, "A $3 \mathrm{GHz}$ Fractional All-Digital PLL With a $1.8 \mathrm{MHz}$ Bandwidth Implementing Spur Reduction Techniques," IEEE J. Solid-State Circ., vol. 44, no. 3, pp. 824-834, Mar. 2009.

[7] T. Rahkonen, J. Kostamovaara, "The use of stabilized CMOS delay line for the digitization of short time intervals," IEEE J. Solid-State Circ., vol. 28, pp. 887-894, Aug. 1993.

[8] J. Kalisz, "Review of methods for time interval measurements with picosecond resolution," Metrologia, vol. 41, no. 1, pp. 1732, Feb. 2004.

[9] P. Dudek, S. Szczepanski, J. V. Hatfield, "A High-Resolution CMOS Time-to-Digital Converter Utilizing a Vernier Delay Line," IEEE Trans On Solid-State Circ., vol. 35, no. 2, Feb. 2000.

[10] B. Helal, M. Straayer, M. Perrott, "A Low Jitter 1.6 GHz Multiplying DLL Utilizing a Scrambling Time-to-Digital Converter and Digital Correlation," VLSI Symp. Dig. Tech. Papers, pp. 166-167, June 2007.

[11] M. Z. Straayer, M. H. Perrott, "An efficient high-resolution 11-bit noise-shaping multipath gated ring oscillator TDC," Symposium on VLSI Circuits Digest of Technical Papers, pp. 82-83, Jun. 2008.

[12] F. Brandonisio, F. Maloberti, "An All-Digital PLL with a First Order Noise Shaping Time-to-Digital Converter," Proc. of 2010 IEEE International Symposium on Circuits and Systems (ISCAS), pp. 241-244, 2010.

[13] R. Schreier, G. C. Temes, "Understanding Delta-Sigma Data Converters,", Wiley-IEEE Press, pp. 42-49, Nov. 2004. 